\title{
When Should We Stop Scanning Older Patients with Vestibular Schwannomas?
}

\author{
Daniele Borsetto $^{1, *}$ Juliette Gair ${ }^{2, *}$ Olivia Kenyon ${ }^{2}$ Tilak Das ${ }^{4}$ Neil Donnelly ${ }^{1}$ Patrick Axon ${ }^{1}$ \\ Robert Macfarlane $^{3}$ Richard Mannion ${ }^{3}$ Daniel Scoffings ${ }^{4}$ Manohar Bance ${ }^{1} \quad$ James Tysome ${ }^{1}$
}

1 Department of Skull Base Surgery, Addenbrooke's Hospital,
Cambridge University Hospitals, Cambridge, United Kingdom
2 Department of Ear, Nose \& Throat, Addenbrooke's Hospital,
Cambridge University Hospitals, Cambridge, United Kingdom
${ }^{3}$ Department of Neurosurgery, Addenbrooke's Hospital,
Cambridge University Hospitals, Cambridge, United Kingdom
${ }^{4}$ Department of Radiology, Addenbrooke's Hospital,
Cambridge University Hospitals, Cambridge, United Kingdom

J Neurol Surg B 2019;80:333-337.

\begin{abstract}
Address for correspondence Daniele Borsetto, MD, Department of Skull Base Surgery, Addenbrooke's Hospital, Cambridge University Hospitals, Cambridge Biomedical Campus, Hills Road, Cambridge CB2 2QQ, United Kingdom (e-mail: daniele.borsetto@gmail.com).
\end{abstract}

\begin{abstract}
Keywords

- vestibular schwannoma

- acoustic neuroma

- surveillance

- observation

- elderly

Objective Observation is a well-accepted management for small- to medium-sized vestibular schwannomas (VSs). Although there are good data on the natural history of this disease within adults, no studies have looked specifically at those aged over 70 years. Thus, there is a need for a surveillance protocol to determine if and when we can stop imaging safely patients aged 70 years and over with a new diagnosis of VSs when managed with observation.

Design Over a 13-year period, we retrospectively analyzed all skull base unit patients with a sporadic unilateral VSs managed with an imaging surveillance protocol. All data were collected prospectively with a minimum follow-up of 5 years.

Setting Tertiary referral skull base unit

Participants Patients aged 70 years and over with sporadic VSs at diagnosis

Main Outcome Measures Main outcome measures

Results A total of 112 patients met inclusion criteria. The median age at diagnosis was 74 years (range: 70-87 years). The mean follow-up was 82 months (range: 60-144). The size of the VSs at diagnosis was intracanalicular (IC) in $46 \%$, small in $41 \%$, medium in $12 \%$, and large in $2 \%$.

Growth was more likely where tumors were extracanalicular (EC) rather than IC at presentation $(p=0.036)$ and within the first 18 months after diagnosis $(p<0.001)$. Twenty-nine percent of VSs displayed growth (6\% continued surveillance, $23 \%$ received active treatment). Good hearing at diagnosis did not predict tumor stability for IC or EC tumors ( $p=0.162$ and $p=0.536)$.

Conclusions Since no VSs grew after 42 months from diagnosis, our data support an initial magnetic resonance imaging (MRI) at 6 months after diagnosis followed by an annual MRI for 3 years. At this point, consideration could be given to discussing discontinuation or further imaging with patients.
\end{abstract}

The first two authors contributed equally to this work.

received

May 24, 2018

accepted after revision

November 13, 2018

published online

December 27, 2018
(C) 2019 Georg Thieme Verlag KG Stuttgart · New York
DOI https://doi.org/

10.1055/s-0038-1676820. ISSN 2193-6331. 


\section{Introduction}

Vestibular schwannomas (VSs) are rare benign tumors of the vestibular nerve. Their intracranial location can cause significant morbidity. For larger tumors, surgery or radiotherapy remains the preferred treatment options, but for smallto medium-sized tumors, conservative management with a "watch, wait, and rescan" (WWR) protocol using serial magnetic resonance imaging (MRI) has been adopted as the preferred initial strategy by most skull base centers. ${ }^{1}$ Although much data have been collected detailing the natural history of VSs managed conservatively, it is not yet clear at what stage it is safe or reasonable to stop imaging patients.

The incidence of VSs is less than 20 tumors per million per year, with an average age of diagnosis of 58 years. ${ }^{2-4}$ However, the increasing use of MRI of the head or internal auditory meatus (IAM) has resulted in the discovery of many incidental VS, in addition to those found specifically after imaging for unilateral hearing loss or tinnitus. In our unit, an increasing number of patients are being given a new diagnosis of VSs when aged at least 70 years. While many patients aged 70 years are medically well and fit for treatment, it is also true that as age increases, medical comorbidities make it increasingly less likely that patients are fit enough for treatment, and, therefore, there may be little logic to continuing surveillance in some patients. In this paper, we present a service evaluation of patients initially presenting at the age of 70 or older with a unilateral VSs who have been initially managed conservatively to derive data about if and when these tumors grow, which facilitates decisions regarding safety in ceasing monitoring of these tumors.

\section{Methods}

The study has been conducted following the STROBE (Strengthening the Reporting of Observational Studies in Epidemiology) guidelines for reporting observational studies.

All patients aged 70 years or over with a sporadic unilateral VSs at the date of diagnosis were identified through a retrospective clinic record review. All data had been prospectively collected from our database of patients with sporadic unilateral VSs managed in our department's skull base unit since 2005 . Only those managed initially with conservative management (WWR) on our standard serial imaging protocol with at least 5 years of follow-up were included to assess tumor growth.

We excluded patients treated with primary surgery or radiotherapy, those who had a diagnosis of neurofibromatosis type 2, and those followed up using computed tomography (CT) imaging rather than MRI.

All patients were initially discussed at our departmental skull base multidisciplinary meeting and management options discussed with the patient in the clinic prior to placing them on a WWR protocol. Our unit policy is, on the whole, to recommend conservative management for patients presenting with smaller tumors with no significant indentation of the brain stem, although all options are discussed and offered to patients so that they can make an informed personal choice regarding management of their tumor.

All patients underwent radiological assessment using MRI. Measurement of the tumor size was taken at the maximum diameter in the cerebellopontine angle (CPA) not including the intracanalicular (IC) portion in accordance with the Committee on Hearing and Equilibrium guidelines. ${ }^{5}$ Tumor size was classified as IC, small ( $\leq 15 \mathrm{~mm}$ in the CPA), medium $(15-25 \mathrm{~mm})$, or large $(>25 \mathrm{~mm})$. Surveillance imaging took place at 6 months after the initial MRI, then annually for 3 years, then every 2 years for 6 years, and then moving to 3-year imaging after 10 years of follow-up as per our standard departmental VS imaging protocol. The preferred MRI protocol was fine slices of the IAMs using T1weighted gadolinium-enhanced MRI; however, in some cases, particularly where intravenous contrast was contraindicated, fast spin echo T2 high-definition volume-acquisition scans (FIESTAs) were used.

An independent measurement of tumor size and growth was undertaken by the authors (D. B., O. K., J. G.) and a consultant neuroradiologist. Digitized scans were examined using the Picture Archiving and Communications System, which has been in use in our department since 2005, and the software used for size assessment was Centricity Enterprise Web version 3.0.

Tumor size was assessed using linear measurements of the maximal diameter in the axial plane. If a tumor demonstrated growth (defined as an increase of at least $2 \mathrm{~mm}$ per year in maximal diameter measured from the initial diagnostic scan), the case was rediscussed in our multidisciplinary meeting, and the management options were discussed with the patient, including further conservative management, radiotherapy, or surgery depending on tumor size and patient factors including hearing status, fitness for treatment, and patient preference.

Tumor size, localization, IAC expansion, and growth pattern, as well as age were compared with speech discrimination score (SDS) at diagnosis; speech audiometry was performed in silence using standardized word lists read from a $C D$, and the SDS was determined by the number of words correctly repeated (in percent) at the most comfortable sound pressure level according to the masking rules.

The hearing was classified according to the modified word recognition scoring (mWRS): class 0 designates patients with $100 \%$ speech discrimination, class 1 designates SDS between 99 and $70 \%$, class 2 designates SDS between 69 and 50\%, class 3 designates SDS between 49 and 1\%, and class 4 designates SDS of $0 \% .^{3}$

\section{Results}

\section{Patients Age, Tumor Size, and Management}

The study identified 218 patients aged 70 or older at the time of the first diagnosis between 2005 and 2012. Of these, 112 patients met the inclusion criteria.

In our cohort of 112 patients, the median age at diagnosis was 74 years (range: $70-87$ years); $48 \%$ presented at age $\geq 75$ years and $12 \%$ at $\geq 80$ years. The mean follow-up was 82 months (range: $60-144$ ). The size of the VS at diagnosis 
was IC in $46 \%$ ( 51 tumors), small in $41 \%$ (46 tumors), medium in $12 \%$ ( 13 tumors), and large in $2 \%$ ( 2 tumors).

Within the total cohort of 112 patients, the subsequent management strategy for the WWR cohort for the following 5 years is shown below:

- Seventy-nine patients (71\%) displayed no growth for the entire follow-up period. This consisted of 41 IC, 30 small, and 8 medium VSs.

- Thirty-three patients (29\%) showed a radiological growth. These were 10 IC, 16 small, 5 medium, and 2 large VSs:

- Eight patients (6\%) continued on a WWR policy despite radiological growth. Tumor size was 3 IC, 1 small, 3 medium, and 1 large VSs. No conversion to radiotherapy or surgery was required due to the lack of extension into the CPA despite growth, the slow rate of growth, patient choice or high risk of treatment in a patient with large VS, multiple comorbidities, and few symptoms.

- Twenty-five patients (23\%) converted to active treatment. Tumor size was 7 IC, 15 small, 2 medium, and 1 large VSs. All patients who converted to active treatment had tumors displaying growth, and the median time before conversion to radiotherapy or surgery was 28 months (range: 6-60 months). Of those who had active treatment:

1. Twenty-two patients (88\%) underwent radiotherapy ( 14 stereotactic radiosurgery and 8 fractionated radiotherapy) as active treatment.

2. Three underwent surgery (12\%): two through a translabyrinthine approach with total removal of the VS; in one case, a retrosigmoid approach was planned with subtotal removal of the tumor due to comorbidities with residual disease stable after 5 years of follow-up.

\section{Hearing}

Hearing data in relation to the VS class size are presented in - Table 1.

IC and small VSs were more likely to have good hearing at diagnosis compared with medium and large VSs ( $p<0.001)$. However, good hearing ( $m$ WRS class 0 and 1 ) at diagnosis did not predict tumor stability for either the IC or extracanalicular (EC) tumors ( $p=0.162$ and $p=0.536$ ).

\section{Tumor Growth}

The likelihood of growth did not change with increasing age (70-74, 75-79, $\geq 80[p=0.90])$. No cases of new radiological growth were seen after 42 months of follow-up (-Fig. 1). Thirty-three tumors (29\%) showed radiological growth during the follow-up period, with growth more likely where tumors were EC at presentation when compared with IC (39 and $18 \%$ respectively; $p=0.036)$ and within the first 18 months after diagnosis $(p<0.0001)$. For tumors that showed growth, the mean annual growth rate was $2.04 \pm 2.29 \mathrm{~mm}$ per year. There was similar growth for IC and EC tumors $(2.10 \pm 2.77$ and $1.70 \pm 2.06 \mathrm{~mm}$ per year, respectively; $p=0.277$ ). Of the EC tumors, $37 \%$ of small tumors and $54 \%$ of medium tumors showed growth.

\section{Discussion}

Office for National Statistics' National Population Projections suggest that the United Kingdom population aged 85 and over is set to double from 1.6 million in mid-2016 to 3.2 million by mid-2041. The number of people of pensionable age (over 67) is forecast to increase from 12.4 million to 16.3 million by 2041.6 Current treatment guidelines for managing VSs are based on standard protocols that may not necessarily take into account patient performance status, comorbidities, or whether the natural history of VS growth is different in older patients. Previous studies in older patients and VSs have largely been small with heterogeneous follow-up. ${ }^{7} \mathrm{~A}$ further consideration in the management of this age group is the impact of on-going follow-up with MRI or discontinuation on the patient and their quality of life.

Of note in this study was the pattern of growth exhibited by the tumors over time. Of the tumors that grew, $90 \%$ grew during the first 30 months after diagnosis. However, our cohort did not display any initial growth after $3 \frac{1}{2}$ years (42 months), implying that the risk of growth is low after this time period in this age group.

Age alone is not a priori a reason to deny a patient skull base surgery or radiotherapy. In addition, well-selected patients may experience improved quality of life following treatment.

Imaging cost, the time and cost to the patient for attending the scan (which may be far from home if at a specialist skull bass unit), and the psychological stress of screening and

Table 1 mWRS class distribution in relation to tumor size of the whole cohort

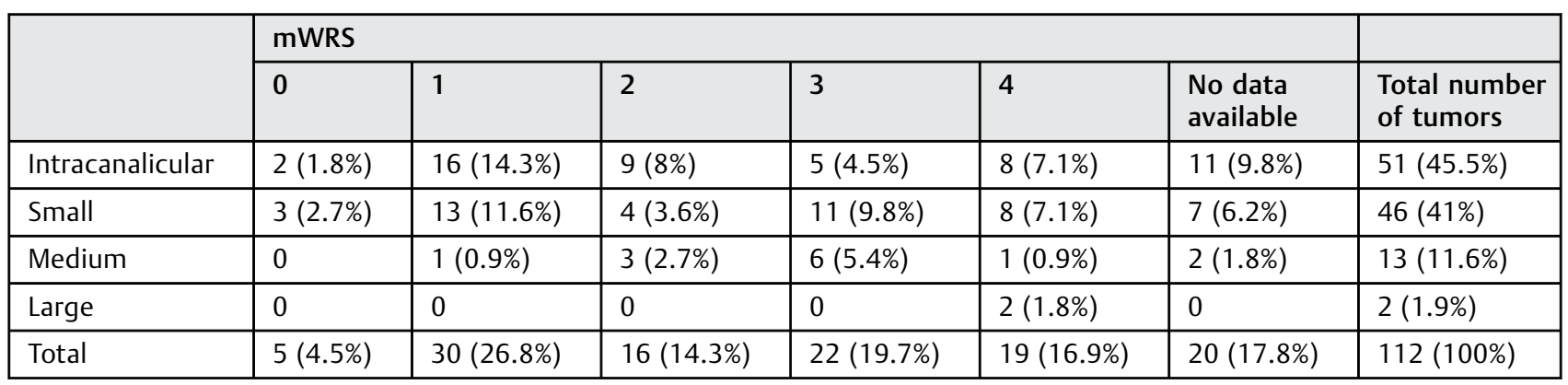

Abbreviation: mWRS, modified word recognition score. 


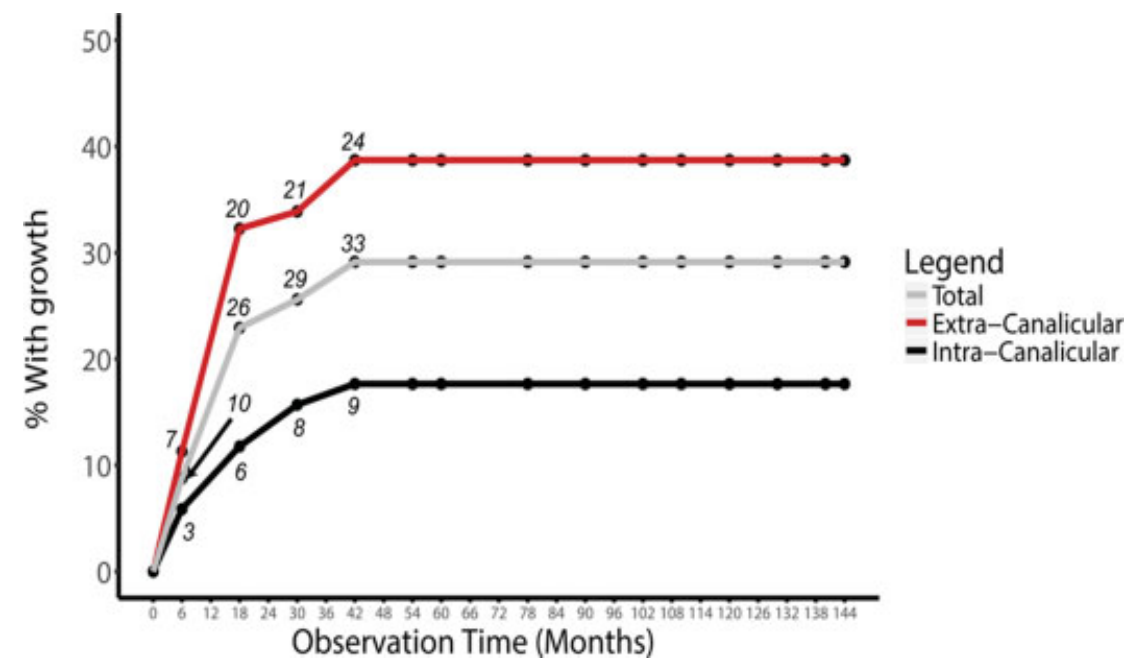

Fig. 1 Nelson-Aalen curve of vestibular schwannoma growth.

awaiting results is important and may impact the decision to continue to scan older patients. This may be even more pertinent in patients with impaired health and other comorbidities, when life expectancy is likely to be conditioned by medical comorbidities, and therefore where continued surveillance is less likely to represent benefit.

Despite the experiences described by some other centers, where older patients had a lower chance of tumor growth occurring, ${ }^{7}$ in our study, age in itself was not a predictor of tumor growth, and the only important factor was tumor size at presentation.

The topic of particular interest in detailing the natural history of VSs in older patients from diagnosis is a review of the long-term risk of tumor growth. The risk of growth after 5 years of follow-up ranges from $0^{3}$ to $7 \%,{ }^{8}$ with some recent studies having demonstrated tumor growth after many years of follow-up. ${ }^{9-12}$ These studies recommend continuing a lifelong policy of surveillance imaging, albeit at a reduced interval frequency for stable tumors. However, these papers have not specifically focused on the older patient age group, and our data suggest that a policy of discontinuation of imaging surveillance after 5 years tumor stability can be considered in this group.

If one looks only at the IC cohort, only $14 \%$ of these tumors progressed to EC extension during the follow-up period. Failure of conservative management with IC tumors is reported at $15 \%,{ }^{13}$ which corresponds approximately with the number of tumors that grow enough to require conversion to treatment.

It should be noted, however, in the Danish study undertaken by Kirchmann et $\mathrm{al}^{13}$ that $37 \%$ of IC tumors grew, which is double that of the growth rate seen in our review of those over 70 years of age.

EC tumors were less suitable for surveillance, with $38.7 \%$ expanding further into the CPA during follow-up and likely requiring active treatment of some kind.

While our data show that no tumors grew in this cohort after 42 months, we also did not find any age-related decreased probability of growth. Hence, it is possible that some of these tumors (such as tumors in younger age groups) may also grow with time, given longer follow-up or a larger cohort, as has been shown in other nonage-segregated studies. ${ }^{11,12}$ We also do not have data at 10 or 20 years, and therefore cannot say with certainty that the risk of growth later is zero but only that it is likely to be low. To change recommendations in the elderly requires either a documented change in the biological behavior of tumors in older adults or a change in the utility of treatment when balancing risks and rewards of treatment versus nontreatment. For instance, one consideration would be if there was an increased incidence of treatment side effects or their functional impact with aging. This may happen if there are existing balance problems, for instance, (more likely in the elderly) which may be exacerbated with both surgical or radiotherapy treatment ${ }^{14}$ and lead to loss of independence. Also, because of decreased remaining life expectancy with aging, there is simply less time to accrue bad outcomes from the underlying disease or indeed from its treatment (e.g., malignant change from radiotherapy). Additionally, because other comorbidities are more prevalent with aging, the relative risk of death or new morbidity is higher from other existing conditions in a younger population. Given these complex calculations, there has to be a more nuanced discussion in the older age group that takes into account the change in the relative weighting of the factors discussed above from a younger population group and is more individualized based on the patients age, comorbidities, and life expectancy; effects of treatment of the patient's independence and quality of life; and risks of long-term side effects from treatment. We think the following treatment recommendations are reasonable:

- For small IC tumors in patients with shortened life expectancy (e.g., less than 3-5 years), no further scanning is recommended.

- For intermediate risk tumors (EC tumors, relatively long life expectancy), discussion with patients regarding no further scanning or long interval scanning (e.g., every 5 years) is recommended. 
- For higher risk tumors (any tumor with early signs of raised intracranial pressure, size greater than $2.5 \mathrm{~cm}$, large cystic components, complex symptoms, long life expectancy, and health), consider scanning at modified intervals in the younger cohort (e.g., every 3 years or so).

Of course, with the myriad of other factors that come into play in each individual patient, these may have to be modified on a case-by-case basis.

If treatment is needed, older patients are likely to tolerate radiotherapy for VSs better than microsurgery, and this is often the chosen treatment option when these tumors are treated, but there are little age-specific data on the radiation-related side effects or control rate in older versus younger patients.

The weaknesses of this study mainly concern the hearing data, which was generally not available, and the relatively small cohort of patients due to a single-center experience. The use of linear rather than volumetric measurement may be considered as another limit as volumetric measurements may be able to more clearly identify. In addition, we did not have much data over 10 years of follow-up.

On the other hand, the main strengths of this study lie in a minimum follow-up of all patients of 5 years and in the very homogeneous population considered. This is the first study in literature on observation in the elderly population, and the key clinical factor to consider in the over 70 group when analyzing the risks and benefits of continued or discontinued surveillance is that no VSs grew after 42 months from diagnosis in our cohort, but we clearly need longer followup and larger cohorts to truly determine the risks in this population to understand the true risks of no follow-up.

\section{Conclusion}

Our review of the patient cohort presenting initially aged 70 or older reveals that all patients demonstrating initial tumor growth did so within 42 months after diagnosis. Since the majority of our cohort had small- and medium-sized tumors, our data support the introduction of a surveillance protocol of MRI 6 months after initial diagnosis followed by an annual MRI for 3 years, at which point consideration can be given to discussing stopping further imaging with patients based on their preference, performance status, and comorbidities.

Note

This study was presented at the Annual Meeting of the British Skull Base Society, Liverpool, United Kingdom, January 25-26, 2018.
Funding

None.

Conflict of Interest

None.

\section{References}

1 Stangerup SE, Caye-Thomasen P, Tos M, Thomsen J. The natural history of vestibular schwannoma. Otol Neurotol 2006;27(04): 547-552

2 Caye-Thomasen P, Dethloff T, Hansen S, Stangerup SE, Thomsen J. Hearing in patients with intracanalicular vestibular schwannomas. Audiol Neurotol 2007;12(01):1-12

3 Stangerup SE, Caye-Thomasen P. Epidemiology and natural history of vestibular schwannomas. Otolaryngol Clin North Am 2012;45(02):257-268, vii

4 Stangerup SE, Tos M, Thomsen J, Caye-Thomasen P. True incidence of vestibular schwannoma? Neurosurgery 2010;67(05):1335-1340, discussion 1340

5 Committee on Hearing and Equilibrium guidelines for the evaluation of hearing preservation in acoustic neuroma (vestibular schwannoma). American Academy of Otolaryngology-Head and Neck Surgery Foundation, INC. Otolaryngol Head Neck Surg 1995; 113(03):179-180

6 Office for National Statistics. National Population Projections: 2016-based statistical bulletin. www.ons.gov.uk. Accessed October 26, 2017

7 Roehm PC, Gantz BJ. Management of acoustic neuromas in patients 65 years or older. Otol Neurotol 2007;28(05):708-714

8 Moffat DA, Kasbekar A, Axon PR, Lloyd SK. Growth characteristics of vestibular schwannomas. Otol Neurotol 2012;33(06):1053-1058

9 Bakkouri WE, Kania RE, Guichard JP, Lot G, Herman P, Huy PT. Conservative management of 386 cases of unilateral vestibular schwannoma: tumor growth and consequences for treatment. J Neurosurg 2009;110(04):662-669

10 Patnaik U, Prasad SC, Tutar H, Giannuzzi AL, Russo A, Sanna M. The long-term outcomes of wait-and-scan and the role of radiotherapy in the management of vestibular schwannomas. Otol Neurotol 2015;36(04):638-646

11 Hunter JB, Francis DO, O'Connell BP, et al. Single institutional experience with observing 564 vestibular schwannomas: factors associated with tumor growth. Otol Neurotol 2016;37(10): $1630-1636$

12 Lees KA, Tombers NM, Link MJ, et al. Natural history of sporadic vestibular schwannoma: a volumetric study of tumor growth. Otolaryngol Head Neck Surg 2018;159(03):535-542

13 Kirchmann M, Karnov K, Hansen S, Dethloff T, Stangerup SE, CayeThomasen P. Ten-year follow-up on tumor growth and hearing in patients observed with an intracanalicular vestibular schwannoma. Neurosurgery 2017;80(01):49-56

14 Tuleasca C, George M, Faouzi M, et al. Acute clinical adverse radiation effects after Gamma Knife surgery for vestibular schwannomas. J Neurosurg 2016;125(Suppl 1):73-82 